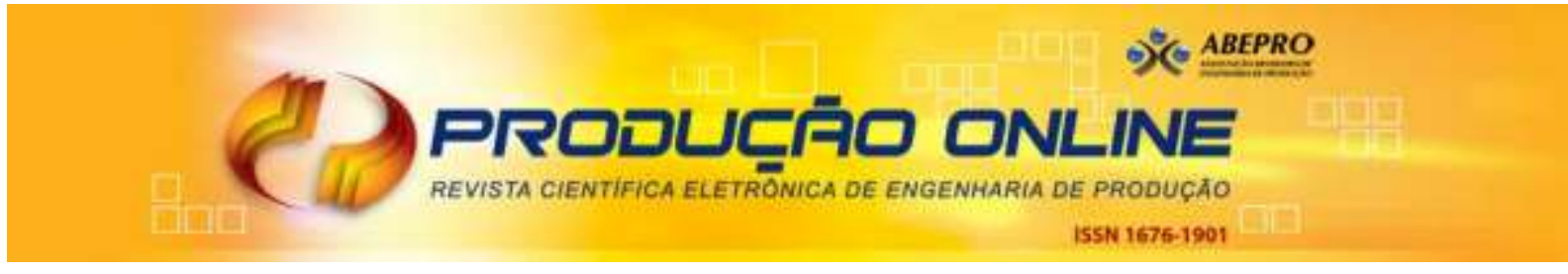

\title{
SIMULAÇÃO COMPUTACIONAL PARA AVALIAÇÃO DO IMPACTO AMBIENTAL GERADO PELA TECNOLOGIA DE INFORMAÇÃO
}

\section{COMPUTER SIMULATION FOR ASSESSMENT OF THE ENVIRONMENTAL IMPACT GENERATED BY THE INFORMATION TECHNOLOGY}

\author{
Giana Lucca Kroth*E-mail: gilucca@gmail.com \\ Eugênio de Oliveira Simonetto*E-mail: eosimonetto@ufsm.br \\ Mauri Leodir Lobler* E-mail: lobler@ccsh.ufsm.br \\ Juçara Salette Gubiani* E-mail: jsgubiani@gmail.com \\ UnHee Schiefelbein* E-mail: peace.unhee@gmail.com \\ *Universidade Federal de Santa Maria (UFSM), Santa Maria, RS
}

\begin{abstract}
Resumo: Em um processo irreversível, pessoas e empresas utilizam as Tecnologias de Informação (TI) em diferentes atividades no dia a dia. Essa demanda leva a indústria de tecnologia a uma busca incessante pela inovação e o resultado é um ciclo de melhorias, novidades e o consequente aumento do descarte desses componentes no meio ambiente bem como o aumento do consumo de energia e a emissão de $\mathrm{CO}^{2}$ na atmosfera. Nesse contexto, a TI Verde surge como uma alternativa para minimizar os efeitos nocivos ao meio ambiente provocados pela fabricação, uso intensivo e descarte da TI. Uma das barreiras que levam as empresas a adiarem a adoção de práticas da TI Verde é o custo envolvido na implantação e a não garantia do retorno esperado. Assim, fazendo uso da própria TI, através da metodologia de Dinâmica de Sistemas e do software Vensim (VENTANA SYSTEMS, 2015), este trabalho buscou simular a redução do impacto ambiental que a implantação de práticas da TI Verde, tais como a virtualização de servidores e o desligamento dos equipamentos quando não estão em uso, podem trazer, ao longo de dez anos, em uma Unidade de TI de uma universidade. Os resultados demonstram que, com a adoção de práticas simples, é possível atingir uma redução de até $62 \%$ no consumo de energia e emissão de $\mathrm{CO}^{2}$.
\end{abstract}

Palavras-Chave: Tecnologia da Informação. TI Verde. Modelagem. Simulação Computacional. Dinâmica de Sistemas.

\begin{abstract}
In an irreversible process, people and businesses use Information Technology (IT) in different activities on a daily basis. This demand leads the technology industry to a relentless pursuit of innovation and the result is a cycle of improvements, innovations and the consequent increase in the disposal of these components into the environment as well as increased energy consumption and the emission of $\mathrm{CO}^{2}$ in the atmosphere. In this context, the Green IT comes up as an alternative to minimize the harmful effects to the environment caused by the manufacturing, intensive use and disposal of IT. One of the barriers that lead companies to delay the adoption of Green IT practices is the cost involved in deploying it and that there are no return guarantee. Thus, making use of IT itself through the system dynamics methodology and Vensim software (VENTANA SYSTEMS, 2015), this study aimed to simulate the reducing of the environmental impact that the implementation of Green IT practices, such as server virtualization and switching off equipment when not in use, can bring over ten years in an IT unit of a university. The results demonstrate that, with very simple practices adoption, it is possible to get a reduction of up to $62 \%$ in energy consumption and emission of $\mathrm{CO}^{2}$.
\end{abstract}

Keywords: Information Technology. Green IT. Modeling. Computer Simulation. System Dynamics. 


\section{INTRODUÇÃO}

$\mathrm{Na}$ atualidade, a cada dia a sociedade demanda mais recursos providos pela Tecnologia de Informação (TI). Em um processo irreversível, pessoas e empresas utilizam o computador em diferentes atividades no dia a dia. Essa demanda leva a indústria a uma busca incessante pela inovação e o resultado é um ciclo de melhorias e novidades a todo o momento, fazendo com que as ferramentas tecnológicas se tornem ultrapassadas em pouquíssimo tempo.

É nesse contexto que a TI Verde surge como uma alternativa sustentável, trazendo um conjunto de políticas, pesquisas, produtos e práticas que buscam minimizar os efeitos danosos ao meio ambiente, provocados pelo uso intensivo da tecnologia da informação (SALLES ET AL, 2013), considerando desde o descarte correto do lixo eletrônico e a utilização de matéria prima e substâncias menos tóxicas na fabricação dos equipamentos, até o consumo de energia e a emissão de gás carbônico (CO'), principal agente do aquecimento global (JAYO; VALENTE, 2010).

De acordo com um estudo da Consultoria Gartner de 2007, a quantidade de $\mathrm{CO}^{2}$ emitida pelos equipamentos de $\mathrm{Tl}$ em todo mundo já é comparável com a quantidade emitida pela frota mundial de aviões, sendo que os datacenters são responsáveis por $23 \%$ da emissão de gases de toda $\mathrm{TI}$, enquanto o uso de PCs e monitores atingem 40\% (LUNARDI ET AL, 2011). Ainda, segundo Jayo e Valente (2010), apenas $25 \%$ das emissões são gerados na produção de computadores e demais equipamentos, enquanto os $75 \%$ restantes são resultantes da utilização dos mesmos. Dessa forma, questões relacionadas à sustentabilidade ambiental tornam-se cada vez mais importantes, tanto na pesquisa científica como na prática das organizações (DAO ET AL, 2011 apud SALLES ET AL, 2013).

Segundo Salles et al (2013) as dimensões econômica e legal são as que mais influenciam ou motivam a adoção de práticas de TI Verde e as organizações apenas passarão a adotar práticas verdes na gestão da Tl, a partir do momento que identificarem benefícios financeiros e operacionais com a adoção. Porém, a adoção destas práticas verdes, tais como aquisição de novos equipamentos que tenham um consumo menor de energia, alterações no layout dos data centers, digitalização de documentos, entre outras tantas, possuem um custo bastante elevado de implantação, o que acaba sendo uma barreira para os gestores, pois não há uma resposta concreta dos reais benefícios que elas podem trazer.

Nesse contexto, a simulação computacional desempenha um papel fundamental, pois permite demonstrar, através de modelos abstratos, os resultados reais que podem ser obtidos modificando-se algumas variáveis do processo. Assim, esse artigo tem como objetivo principal apresentar o desenvolvimento e validação de um modelo de simulação computacional que permita avaliar o impacto ambiental, considerando a emissão de $\mathrm{CO}^{2}$ e o consumo de energia, de uma Unidade de TI de uma universidade, com e sem a adoção de práticas da TI Verde.

O artigo está organizado da seguinte forma: a primeira seção apresenta o problema e os objetivos; a segunda seção discute o embasamento teórico do artigo, 
apresentando em maior profundidade o tema da TI Verde e a definição de simulação computacional; a terceira seção apresenta a metodologia utilizada no estudo; a quarta seção apresenta a definição do modelo e a construção do modelo matemático, enquanto a quinta seção descreve o modelo desenvolvido e os resultados dos experimentos com os diferentes cenários. Por fim a sexta seção conclui e apresenta as considerações finais do estudo e as referencias bibliográficas são identificadas na última seção.

\section{REFERENCIAL TEÓRICO}

Nesta seção serão apresentados os temas TI Verde e Simulação Computacional, que se relacionam neste trabalho com o intuito de auxiliar o processo de tomada de decisão pela adoção ou não de práticas sustentáveis relacionadas ao uso da TI.

\subsection{TI Verde}

A TI Verde pode ser encarada como uma nova visão que algumas empresas estão adotando, com o objetivo de minimizar os impactos da TI no meio ambiente. De forma mais abrangente, podemos citar:

\footnotetext{
A TI é parte do problema ambiental, e pode ser parte da solução. A TI Verde é um imperativo tanto econômico quanto ambiental. Tornar a TI mais verde é, e vai continuar sendo, uma necessidade e não uma opção. A TI Verde representa uma mudança dramática na indústria da TI. [...] Os desafios da TI Verde são enormes; no entanto, pesquisas recentes indicam que a indústria da TI possui vontade e convicção para enfrentar de frente os nossos problemas ambientais (MURUGESAN, 2008 tradução nossa).
}

Murugesan (2008) indica que, para compreender melhor o impacto da TI no ambiente, deve-se ter adotar uma abordagem holística e avaliar quatro momentos distintos: (a) o projeto da $\mathrm{TI}$ - projetando de forma mais econômica os computadores, servidores, periféricos, etc; (b) a fabricação da $\mathrm{TI}$ - de forma a agredir o mínimo possível o meio ambiente durante o processo de fabricação; (c) o uso da $\mathrm{TI}$ - reduzindo o consumo de energia na utilização dos computadores; e (d) o descarte da TI - reutilizando ou reciclando antigos computadores.

A redução do consumo de energia elétrica é o maior obstáculo dos projetos de TI verde, já que boa parte dos gastos em um ambiente tecnológico tem como fator principal a energia elétrica. Murugesan (2008) destaca técnicas simples que podem auxiliar neste processo, tais como: desligar os computadores quando não estiverem em uso, habilitar o sistema de gerenciamento automático de energia dos PCs, usar protetores de tela pretos ao invés de figuras se movendo, entre outros.

No caso dos data centers, além da adoção de tecnologias de refrigeração mais "limpas", uma solução que tem se apresentado como promissora é a chamada virtualização de servidores, uma técnica que permite rodar, simultaneamente, mais 
de um sistema operacional em um único equipamento físico, que diminui a capacidade de processamento ociosa em cada servidor e permite reduzir o tamanho (número de servidores) e, como consequência, o consumo dos data centers. Embora essa inovação tenha sido desenvolvida originalmente para poupar custos com máquinas e espaço físico, ela se revelou vantajosa também para a economia de recursos naturais (UDDIN; RAHMAN, 2010).

O Quadro 1 resume os enfoques de alguns dos estudos utilizados como referência nesta pesquisa, para a categorização das práticas verdes.

Quadro 1 - Categorização das práticas verdes

(continua)

\begin{tabular}{|c|c|c|}
\hline Estudo & Categorização & Detalhes \\
\hline $\begin{array}{l}\text { MURUGESAN, } \\
2008\end{array}$ & $\begin{array}{l}\text { Classificação da abran- } \\
\text { gência das práticas de } \\
\text { TI Verde em } 3 \text { níveis }\end{array}$ & $\begin{array}{l}\text { (a) abordagem incremental - medidas simples, } \\
\text { de baixo ou nenhum custo, preservando a infra- } \\
\text { estrutura atual de TI e com retorno rápido; } \\
\text { (b) abordagem estratégica - São definidas politi- } \\
\text { cas internas com o objetivo de diminuir os riscos } \\
\text { ao meio ambiente e impacto de seus produtos; } \\
\text { (c) abordagem radical verde (Green IT) - mudan- } \\
\text { ças totais em instalações, padronizações de pro- } \\
\text { cessos. }\end{array}$ \\
\hline $\begin{array}{l}\text { LUNARDI ET AL, } \\
2011\end{array}$ & $\begin{array}{l}\text { Classificação das práti- } \\
\text { cas de TI Verde em } 7 \\
\text { categorias }\end{array}$ & $\begin{array}{l}\text { (a) práticas de conscientização, } \\
\text { (b) datacenter verde, } \\
\text { (c) descarte e reciclagem, } \\
\text { (d) fontes alternativas de energia, } \\
\text { (e) hardware, } \\
\text { (f) impressão } \\
\text { (g) software }\end{array}$ \\
\hline DIAS ET AL, 2013 & $\begin{array}{l}\text { Definição de } 6 \text { dimen- } \\
\text { sões para análise da TI } \\
\text { Verde }\end{array}$ & $\begin{array}{l}\text { (a) Política e Conscientização ambiental, } \\
\text { (b) Eficiência energética; Energias renováveis, } \\
\text { (c) Infraestrutura de TI (Green Datacenters, Vir- } \\
\text { tualização, Cloud Computing), } \\
\text { (d) Infraestrutura física verde (Prédios verdes), } \\
\text { (e) Lixo eletrônico, reutilização, reciclagem, Des- } \\
\text { carte, } \\
\text { (f) Seleção de fornecedores e Selos verdes }\end{array}$ \\
\hline $\begin{array}{l}\text { SOUZA; SILVA, } \\
2013\end{array}$ & $\begin{array}{l}\text { Definição de } 4 \text { dimen- } \\
\text { sões analíticas: os } 4 \text { P's } \\
\text { da TI Verde }\end{array}$ & $\begin{array}{l}\text { (a) Postura Verde (Sustentabilidade, Gestão Sus- } \\
\text { tentável, Leis, Normas e Diretrizes e Redução de } \\
\text { Carbono), } \\
\text { (b) Prática Verde (Racionalização de Energia, } \\
\text { Racionalização de Insumos e Materiais e Geren- } \\
\text { ciamento de Insumos), } \\
\text { (c) Política Verde(Aquisição, Substituição de } \\
\text { Ativos e Descarte) e } \\
\text { (d) Produção Verde (Projeto, Fabricação e Tec- } \\
\text { nologia). }\end{array}$ \\
\hline
\end{tabular}


Quadro 2 - Categorização das práticas verdes

(conclusão)

\begin{tabular}{|c|c|c|}
\hline Estudo & Categorização & Detalhes \\
\hline $\begin{array}{l}\text { FARIA ET AL, } \\
2013\end{array}$ & $\begin{array}{l}\text { A partir da análise de } \\
\text { outras publicações defi- } \\
\text { niram } 7 \text { formas de im- } \\
\text { plantar ações verdes } \\
\text { nas empresas }\end{array}$ & $\begin{array}{l}\text { (a) Compra de hardware mais econômico em } \\
\text { termos energéticos; } \\
\text { (b) Consolidação e Virtualização; } \\
\text { (c) Redução de temperatura nos Data Centers; } \\
\text { (d) Uso de thin clients para reduzir uso de ener- } \\
\text { gia; } \\
\text { (e) Reciclagem e descarte de equipamentos; } \\
\text { (f) Economia no consumo de papel; } \\
\text { (g) Conscientização de seus profissionais }\end{array}$ \\
\hline $\begin{array}{l}\text { LUNARDI ET AL, } \\
2014\end{array}$ & $\begin{array}{l}\text { Identificação de } 5 \text { com- } \\
\text { ponentes que influenci- } \\
\text { am a forma como as } \\
\text { organizações abordam } \\
\text { a dimensão ambiental } \\
\text { da sustentabilidade na } \\
\text { área de TI. }\end{array}$ & $\begin{array}{l}\text { (a) Orientação Ambiental (orientações sobre reci- } \\
\text { clagem, desligar equipamentos e economizar } \\
\text { energia) } \\
\text { (b) Ações Sustentáveis (descarte, aquisição de } \\
\text { produtos mais eficientes em termos energéticos, } \\
\text { virtualização) } \\
\text { (c) Monitoramento (dos custos de manutenção, } \\
\text { do desempenho dos equipamentos, das impres- } \\
\text { sões desnecessárias) } \\
\text { (d) Expertise Ambiental (busca por conhecimento } \\
\text { e novidades para economia de energia) } \\
\text { (e) Consciência Socioambiental (estratégias e } \\
\text { políticas) }\end{array}$ \\
\hline
\end{tabular}

Fonte: Elaborado pelos autores

No caso das universidades, Dias et al (2013), identificaram que as práticas de TI Verde utilizadas são basicamente de abordagem incremental, que são medidas simples, de baixo ou nenhum custo, preservando a infraestrutura atual de $\mathrm{Tl}$ e com retorno rápido, segundo definição de Murugesan (2008). Os autores observaram a ausência de investimento em soluções de virtualização, como os datacenters verdes e a computação em nuvem (cloud computing), e concluíram que a disseminação de práticas de TI Verde na maior parte das Instituições de Ensino Superior do Brasil ainda é incipiente, e algumas práticas ainda se encontram apenas em fase de planejamento.

Chou e Chou (2012) definiram que a TI Verde é apenas uma parte do caminho a ser percorrido pelas empresas para alcançarem a sustentabilidade ambiental. Este caminho foi chamado pelos autores de Modelo de Valor da TI Verde ("Green IT value model'), e engloba quatro componentes distintos, porém contínuos: consciência, tradução, compreensão e valor de TI Verde. A primeira etapa indica que uma maior consciência da sustentabilidade ambiental pode motivar os indivíduos e as organizações para procurar pelo valor da TI verde. A transformação dessa consciência em ações de TI Verde seria a segunda etapa do modelo, e a terceira etapa está relacionada com o monitoramento das ações, a definição de planos e estratégias. Por fim, como um resultado dos esforços das três etapas anteriores, é que se poderia dizer que a TI Verde de valor está sendo realizada e que pode trazer sustentabilidade ambiental. 
Baseado nesse conceito de modelo de valor, Chou (2013) identificou os possíveis riscos envolvidos em cada uma das fases e que poderiam afetar os resultados das práticas de TI Verde. Dentre esses riscos estão a dificuldade em se modificar os comportamentos das pessoas da empresa e o pouco esforço por parte dos governos (consciência), a falta de apoio da alta gerencia (tradução), o pouco conhecimento nas métricas da TI Verde e escolha equivocada dos equipamentos para medição do uso de energia (compreensão) e a incapacidade de interpretar os resultados do valor da TI Verde e de comunicação com a sociedade (valor da TI Verde).

Apesar de ainda serem poucos os estudos empíricos na área, no Brasil, alguns esforços estão sendo feitos para criar e validar instrumentos que consigam quantificar os esforços e as abordagens da TI Verde pelas empresas (LUNARDI ET AL, 2015; LUNARDI ET AL, 2014). No âmbito internacional, e com enfoque um pouco diferenciado, o estudo de Cooper e Molla (2014) avaliou o que influencia a assimilação da TI Verde pelas organizações, e relacionou o nível de absorção da TI Verde, isto é, como e quão profundamente as práticas da TI Verde estão incorporadas nas pessoas, no gerenciamento e na infraestrutura de $\mathrm{TI}$, com a teoria contextual e a teoria de capacidade de absorção.

Segundo Murugesan (2008), além da própria TI se tornar verde, ela pode dar suporte para outras iniciativas ambientais, através de ferramentas de apoio à decisão para analisar, modelar e simular o impacto e o risco ambiental. Seriam as aplicações verdes da TI, ou a TI para o verde (FAUCHEUX;NICOLAÏ, 2011). Arnfalk et al (2015) destacam que este seria o efeito indireto da TI, e no caso apresentado pelos autores, os mesmos comprovaram que foi possível atingir uma redução de $10 \%$ nas emissões de gases poluentes com a promoção de reuniões virtuais, reduzindo o número de viagens de negócios para participação de reuniões presenciais.

Ainda assim, existe certa rejeição por parte de algumas empresas, pois as ações de TI Verde podem gerar gastos elevados. Paraíso et al (2009) consideram que "a TI Verde é considerada uma importante força para atenuar o avanço do aquecimento global, porém o grande problema de transformar uma organização em verde seria o custo envolvido nessa mudança".

Boa parte das práticas de sustentabilidade pode ser adotada sem que a saúde financeira da organização seja comprometida, apenas dependendo do esforço e vontade dos funcionários, e do apoio e direcionamento da organização. As práticas ligadas aos datacenters e à substituição de equipamentos obsoletos são demandantes de altos investimentos, porém, aparecem como as práticas cujo retorno financeiro é o mais rápido e, por consequente, as mais impactantes na redução de custos da organização (LUNARDI ET AL, 2011).

Segundo informações do site do SEBRAE (SEBRAE, 2015) e do relatado em Lunardi et al (2011), as empresas que possuem a preocupação ambiental e adotam algumas práticas verdes possuem vantagens econômicas como facilidade de créditos e redução taxas e impostos por parte dos órgãos governamentais. Além disto, há uma melhor imagem frente à sociedade, impulsionando as ações de marketing para atrair aqueles consumidores que têm preferencia por empresas responsáveis soci- 
almente. Brown (2009, apud FARIA ET AL, 2013) considera que, ao aplicar a TI Verde, a sociedade passa a ver a empresa como diferenciada das demais, elevando seus status e colocando-a como referência entre as concorrentes, levando à confiança e preferência de seus clientes.

Neste mesmo contexto, Chuang e Huang (2015) definiram um modelo teórico, comprovado empiricamente, onde o grau de envolvimento das empresas com a sustentabilidade ("business greening") está associado positivamente com o capital de $\mathrm{TI}$ Verde, assim como, o capital de TI Verde influencia positivamente a competitividade da empresa. Por capital de TI verde, os autores entendem a competência e os ativos organizacionais que aplicam conceitos verdes à infraestrutura de $\mathrm{TI}$, à equipe de $\mathrm{TI}$, ao gerenciamento de $\mathrm{Tl}$ e aos relacionamentos. Como resultado, o capital de TI verde não só é capaz de satisfazer a consciência ambiental do consumidor, mas também pode ajudar uma empresa a criar uma vantagem competitiva.

Capital de TI Verde é composto por três dimensões: (1) capital estrutural da TI verde, que se refere à infraestrutura de TI verde, incluindo hardware, software, rede e tecnologia da informação, estabelecido no âmbito do conceito de "tornar-se verde" ("greening"); (2) capital humano da TI verde, que se refere à capacidade e experiência da equipe de $\mathrm{TI}$ em possuir conhecimento profissional verde e uma compreensão de conservação de energia em tecnologia, bem como o desenvolvimento pessoal, com capacidades de TI verde, através de treinamento e educação; e (3) capital relacional da TI verde, referindo-se ao gerenciamento de TI verde e uma relação em que as empresas mantenham melhores relações com os parceiros e usuários com um conceito de proteção ambiental na oferta de produtos e serviços. (CHUANG e HUAND, 2015).

Considerando todo este contexto de preocupação por parte das empresas com os custos envolvidos na adoção de práticas verdes e as considerações de como utilizar a própria TI para o Verde, este trabalho utilizou-se da simulação computacional como forma de prever as reduções de custos que podem ser obtidas com a utilização de algumas práticas verdes.

\subsection{Modelagem e Simulação Computacional}

Um modelo, segundo Pidd (1998), consiste em um mapa daquilo que está ocorrendo, de uma realidade, e do que poderia ser feito sobre ele, isto é, como utilizar o modelo para entender, mudar, gerenciar e controlar parte daquela realidade. Dentre as características e benefícios de um modelo, Ragsdale (2009) destaca que eles são versões simplificadas do objeto ou problema de decisão que oferecem informações necessárias no tempo certo e nos permitem ganhar conhecimento e entendimento sobre o objeto ou problema de decisão investigado. Além disto, o autor destaca que além de ser mais barato analisar problemas de decisão utilizando modelos, em alguns casos, podemos examinar coisas que seriam impossíveis na vida real, como testes com manequins de air bags, por exemplo. 
A modelagem é o processo de criação de modelos; modelo é uma representação simplificada e explícita da realidade com algum propósito definido; e simulação é o processo de manipular o modelo de uma forma dinâmica, como um fluxo de entrada, processamento e saída de algo (ROSSONI, 2006).

Para Rossoni (2006), "a simulação sempre significa a manipulação de um modelo de uma maneira que proporcione uma visão dinâmica, quase cinematográfica da realidade". A simulação computacional consiste na utilização de técnicas computacionais que, por meio de modelos, permitem simular o comportamento do sistema real de interesse e conduzir experimentos com o objetivo de entender o comportamento de um sistema ou avaliar sua operação (NETO ET AL, 2014). Conforme Nascimento et al. (2014), simular um sistema complexo é de suma importância nos dias atuais como alternativa de tomada de decisão, e para isso necessita-se desenvolver um modelo que vise retratar a realidade da melhor forma possível.

Segundo Freitas Filho (2008), a simulação serve para efetuar análises do comportamento do sistema em condições específicas, buscando o encaminhamento de uma solução a um dado problema. $O$ autor destaca ainda que a simulação pode ser usada para prever o estado de um sistema em algum ponto no futuro, baseado nas suposições sobre seu comportamento atual e de como continuará se comportando ao longo do tempo. As razões mais comuns para experimentar-se com modelos simulados são: o sistema real ainda não existe; experimentar com o sistema real é muito caro ou leva muito tempo; experimentar com o sistema real não é apropriado.

Uma das técnicas utilizadas para a simulação computacional é a metodologia de System Dynamics (SD), ou dinâmica de sistemas, que foi desenvolvida durante a década de 1950 pelo engenheiro Jay Forrester, do Instituto Tecnológico de Massachussets (MIT) (FORRESTER, 1961). Dentro da Pesquisa Operacional (PO), a Dinâmica de Sistemas é considerada uma técnica de modelagem soft ou interpretativa, que segundo Pidd (1998) é uma forma de gerar debate e insights a respeito do mundo real através de modelos conceituais e simbólicos, cujo resultado é o processo de aprendizagem, diferente das técnicas hard, ou matemáticas, onde se busca um resultado objetivo e único para um problema.

Por meio de diagramas de estoques (variáveis de estado, acumuladas), fluxos (variáveis de ação que alteram os estoques), auxiliares (definem as equações dos fluxos, modelam as informações) e conectores (representam as inter-relações entre todos os componentes do sistema), tal abordagem contempla a compreensão de fenômenos sistêmicos, relacionando a estrutura de um sistema com o seu comportamento ao longo do tempo, através da geração de cenários que auxiliam o processo de tomada de decisão (PAPPEN; YONENAGA, 2014).

\section{METODOLOGIA}

Por utilizar a Dinâmica de Sistemas, este trabalho está inserido dentro da área de Pesquisa Operacional (PO), que segundo Andrade (2011), é uma metodolo- 
gia administrativa, mas que possui caráter multidisciplinar, pois congrega conhecimentos da Economia, Matemática, Estatística e Computação. Assim, este estudo seguirá as fases descritas por Andrade (2011) como as principais etapas que devem ser cumpridas num trabalho de PO, considerando também que Ragsdale (2009) descreveu estas mesmas etapas como sendo as fases do processo genérico de solução de problemas:

(i) definição do problema: Estudos exploratórios em artigos científicos e manuais de referência sobre as práticas de TI Verde, bem como entrevistas com os técnicos responsáveis pela infraestrutura da Unidade de TI para a identificação das variáveis e restrições envolvidas e para a definição de quais práticas verdes farão parte da simulação;

(ii) construção do modelo de equações: Identificação das variáveis envolvidas e suas relações, definindo o modelo lógico que representa o comportamento do sistema real;

(iii) solução do modelo: Implementação computacional da solução, utilizando-se o simulador Vensim (VENTANA SYSTEMS, 2015) da área de System Dynamics, que permite avaliar o comportamento do sistema ao longo do tempo, demonstrando as consequências da adoção das práticas verdes selecionadas na fase de definição;

(iv) validação do modelo: através da simulação de um experimento, utilizando-se dois cenários: a) o atual sem utilização de nenhuma prática da TI Verde e b) um cenário proposto com a adoção das práticas verdes selecionadas;

(v) implementação dos resultados: nesta fase os dados são apresentados para a unidade de $\mathrm{TI}$, porém a implementação real das práticas fica fora do escopo deste trabalho por ser de competência da universidade.

Nas próximas seções será detalhada cada uma das fases descritas na metodologia, sendo a seção 4 destinada para documentar as etapas de definição do problema e construção do modelo de equações, e a seção 5 para as etapas de solução e validação do modelo.

\section{DEFINIÇÃO DO PROBLEMA E CONSTRUÇÃO DO MODELO DE EQUAÇÕES}

A Unidade de TI selecionada para validar e servir como base para o modelo possui 40 funcionários dedicados e mais aproximadamente 35 estagiários (essa aproximação se deve a grande rotatividade desses), que fazem uso de 80 computadores pessoais (PCs) e de 115 monitores. A utilização destes equipamentos ocorre apenas durante o horário de expediente ( 8 horas/dia), mas é uma prática comum que os mesmos não sejam desligados quando estão em desuso, permanecendo constantemente ligados. Além disto, conta com um data center formado por 48 servidores físicos e 92 servidores virtuais, os quais permanecem ligados e refrigerados 
24 horas, e com um gerador de energia que tem a função de garantir a disponibilidade dos serviços de TI mesmo com falha no fornecimento de energia.

Dois técnicos responsáveis pela manutenção da infraestrutura da unidade foram selecionados para realizar as entrevistas. Após descrição detalhada da infraestrutura, os técnicos definiram que as práticas verdes a terem a sua adoção simulada deveriam ser:

- o aumento na virtualização dos servidores físicos;

- a redução do número de horas que os monitores ficam ligados;

- a redução do número de horas que os computadores pessoais ficam ligados.

O Quadro 2 relaciona as práticas selecionadas com as classificações encontradas na literatura e descritas no Quadro 1.

Quadro 3 - Classificação das práticas selecionadas para a simulação

\begin{tabular}{|c|c|c|}
\hline Prática selecio- & Classificação & Referência \\
\hline \multirow{6}{*}{$\begin{array}{l}\text { Desligar Equipa- } \\
\text { mentos quando } \\
\text { não estiverem em } \\
\text { uso }\end{array}$} & Incremental & MURUGESAN, 2008 \\
\hline & Práticas de conscientização & LUNARDI; FRIO; SALLES, 2011 \\
\hline & $\begin{array}{l}\text { Política e conscientização Ambien- } \\
\text { tal }\end{array}$ & DIAS et al, 201 \\
\hline & Prática Verde & SOUZA; SILVA, 2013 \\
\hline & $\begin{array}{l}\text { Conscientização de seus profissio- } \\
\text { nais }\end{array}$ & $\begin{array}{l}\text { FARIA; MARTINS; SIQUEIRA, } \\
2013\end{array}$ \\
\hline & Orientação Ambiental & LUNARDI; ALVES; SALLES, 2014 \\
\hline \multirow{6}{*}{$\begin{array}{l}\text { Aumentar a virtua- } \\
\text { lização e diminuir a } \\
\text { compra de novos } \\
\text { servidores }\end{array}$} & Estratégica & MURUGESAN, 2008 \\
\hline & Data Center verde & LUNARDI; FRIO; SALLES, 2011 \\
\hline & Infraestrutura de $\mathrm{TI}$ & DIAS et al, 201 \\
\hline & Política Verde & SOUZA; SILVA, 2013 \\
\hline & Consolidação e Virtualização & $\begin{array}{l}\text { FARIA; MARTINS; SIQUEIRA, } \\
2013\end{array}$ \\
\hline & Ações Sustentáveis & LUNARDI; ALVES; SALLES, 2014 \\
\hline
\end{tabular}

Fonte: Elaborado pelos autores

Para verificar os resultados com a adoção de práticas verdes, e validar o modelo construído, foram definidos dois cenários a serem simulados, numa projeção para 10 anos:

- Cenário Atual: considerando a taxa de virtualização atual e que os monitores e PCs ficam ligados por 24 horas;

- Cenário Verde, aumentando a taxa de virtualização dos servidores, e diminuição no tempo que os PCs e os monitores ficam ligados durante o dia.

Ao final das entrevistas, chegou-se ao modelo de equações, descrito na Figura 1, que relata como ocorre o consumo de energia e emissão de $\mathrm{CO}^{2}$. As variáveis que influenciam nos valores finais de emissão de $\mathrm{CO}^{2}$ e consumo de energia com base nas práticas selecionadas, bem como as suas inter-relações com outras variáveis envolvidas no modelo, podem ser descritas da seguinte forma: 
- taxa variável de virtualização de servidores físicos (Taxa de virtualização dos servidores): influencia diretamente no número de servidores mantidos (Servidores Físicos), calculado pelos fluxos de aquisição (Aquisição de servidores) e de descarte (descarte de servidores), ou seja, utilizou-se a fórmula de entrada de servidores variando conforme uma taxa fixa (Taxa de aquisição dos servidores) e a saída de servidores ou por taxa fixa de descarte (Taxa de descarte dos servidores) ou por taxa variável de virtualização, conforme pode ser visualizado nas equações $1,2 \mathrm{e}$ 3 do modelo matemático descrito na Figura 1. O Resultado dessas equações é base para o cálculo do consumo de energia e da emissão de $\mathrm{CO}^{2}$ pelos servidores (Consumo Servidores e Emissão Servidores) conforme equações 4 e 5 do modelo;

Figura 1 - Modelo de Equações da Simulação Desenvolvida

\begin{tabular}{|c|c|}
\hline (1) & Servidores Fisicos $(t)=$ Aquisiçăo de Servidores $(t)$ - Descarte de Servidor es(t) \\
\hline (2) & Aquisiçăo de Servidores $(t)=$ Servidor es Fisicos $(t) *$ Taxa de aquisição de servidores \\
\hline \multirow[t]{2}{*}{ (3) } & Descarte de Servidores $(t)=\left(\right.$ Servidores Fisicos $(t)^{*}$ taxa de virtualizaçăo dos servidores $)+($ Servidores \\
\hline & Fisicos(t)*taxa de descarte dos servidores) \\
\hline (4) & Consumo Servidores $(t)=$ Servidores Fisicos $(t)^{*}$ Média Consumo Servidor \\
\hline (5) & Emissão Servidores $(\mathrm{t})=$ Servidores Fisicos $(\mathrm{t})^{+}$Média de Emissäo Servidor \\
\hline (6) & Monitores $(t)=$ Aquisiçăo Monitores $(t)$-Descarte Monitores $(t)$ \\
\hline (7) & Aquisiçăo Monitores $(t)=$ monitores $(t)^{*}$ taxa aumento número Monitores \\
\hline (8) & Descarte Monitores(t) =monitores(t)*Taxa descarte monitores \\
\hline (9) & Consumo monitores $(t)=$ monitor es $(t)^{*}$ Média Consumo Monitor ${ }^{*}($ Tempo monitor ligado $/ 24)$ \\
\hline$(10)$ & Emissão Monitores $(\mathrm{t})=$ monitores $(\mathrm{t})^{*}$ Média Emissăo Monitor ${ }^{*}\left(\mathrm{Tempo}^{2}\right.$ monitor ligado/24) \\
\hline (11) & $\mathrm{PCs}(\mathrm{t})=$ Aquisiçăo de $\mathrm{PCs}(\mathrm{t})-\mathrm{Descarte}$ de $\mathrm{PCs}(\mathrm{t})$ \\
\hline (12) & Aquisição de $\mathrm{PCs}(\mathrm{t})=\mathrm{PCs}(\mathrm{t})^{*}$ taxa de aumento no numero de $\mathrm{PCs}$ \\
\hline (13) & Descarte de $\mathrm{PCs}(\mathrm{t})=\mathrm{PCs}\left(\mathrm{t}^{*}\right.$ taxa de descarte de PCs \\
\hline (14) & Consumo $\mathrm{PCs}(\mathrm{t})=\mathrm{PCs}(\mathrm{t})^{*}$ Média Consimo $\mathrm{PC}^{*}($ Tempo $\mathrm{Pc}$ Ligado 24$)$ \\
\hline (15) & Emissão $\mathrm{PCs}(\mathrm{t})=\mathrm{PCs}(\mathrm{t})^{*}$ Média Emissão $\mathrm{PC}^{+}(\mathrm{Tempo} \mathrm{Pc}$ Ligado/24) \\
\hline (16) & Consumo de Energia $(t)=$ Consumo Monitores $(t)+$ Consumo $\mathrm{PC}(\mathrm{t})+$ Consumo Servidores $(\mathrm{t})$ \\
\hline (17) & Emissão de CO2(t)= Emisșio Monitores $(t)+$ Emissảo $P C(t)+$ Emissäo Servidores $(t)$ \\
\hline
\end{tabular}

- tempo que os monitores ficam ligados (tempo monitor ligado): influencia diretamente no cálculo do consumo (Consumo monitores) e da emissão de $\mathrm{CO}^{2}$ (Emissão monitores) por parte dos monitores. Além do tempo ligado, há a influência do número de monitores disponíveis (monitores), que é calculado com base nos fluxos de aquisição (aquisição monitores) e de descarte (descarte monitores) e nas taxas médias de aquisição (taxa aumento número monitores) e descarte (taxa descarte monitores) dos mesmos, conforme pode ser visualizado nas expressões $6,7,8,9$ e 10 do modelo; 
- tempo que os PCs ficam ligados (tempo PC ligado): influencia diretamente no cálculo do consumo (Consumo PCs) e da emissão de $\mathrm{CO}^{2}$ (Emissão $\boldsymbol{P C}$ ) por parte dos computadores pessoais. Além do tempo ligado, há a influência do número de PCs disponíveis ( $\boldsymbol{P C s}$ ), que é calculado com base nos fluxos de aquisição (aquisição PCs) e de descarte (descarte PCs) e nas taxas médias de aquisição (taxa de aumento no número PCs) e descarte (taxa de descarte de PCs) dos mesmos, conforme pode ser visualizado nas expressões 11,12,13,14 e 15 do modelo;

- consumo total de energia (consumo de energia) e Emissão total de $\mathrm{CO}^{2}$ (emissão de $\mathrm{CO}^{2}$ ) por parte da unidade de TI: calculados com base nos consumos e emissões de cada um dos três equipamentos avaliados - expressões 16 e 17 do modelo.

\section{SOLUÇÃO E VALIDAÇÃO DO MODELO}

Após a definição do modelo matemático e da definição dos valores das constantes, partiu-se para a terceira etapa definida na metodologia que consiste na implementação do modelo de simulação no software VenSim (VENTANA SYSTEM, 2015), e cujo resultado pode ser visualizado na Figura 2.

Figura 2 - Modelo de Simulação desenvolvido

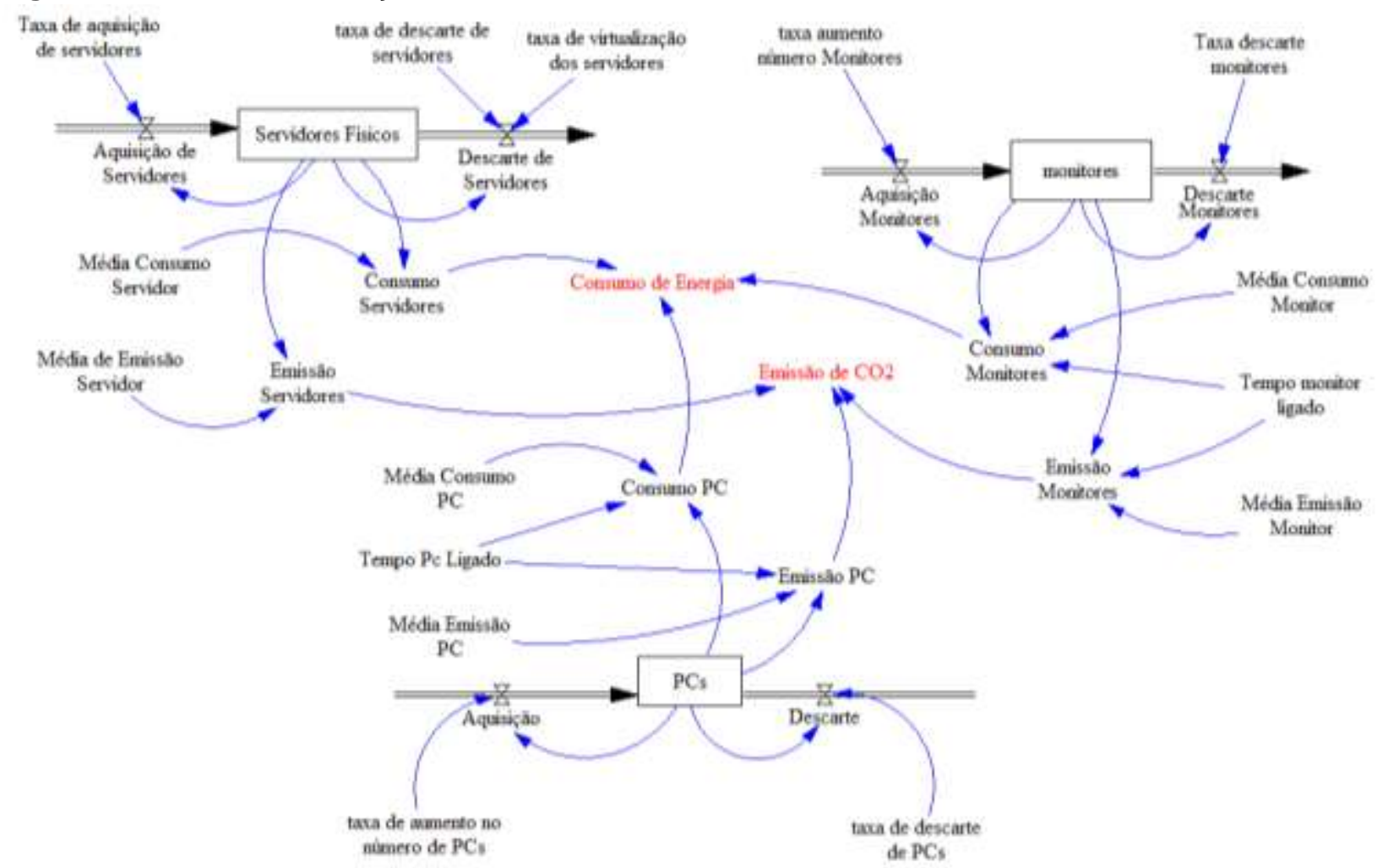

A quarta etapa da metodologia consistiu na validação do modelo e foram selecionados dois tipos de validação de sistemas, descritos por Borenstein e Becker (2000) como Validação Nominal e Análise de Sensibilidade. Segundo os autores, a 
validação Nominal é baseada na opinião de especialistas e tem o objetivo de verificar se o problema está bem formulado e contém as variáveis-chave para a sua representação. Nesta etapa, os técnicos selecionados para as entrevistas verificaram o modelo proposto e atestaram que está de acordo com a realidade.

Numa segunda etapa, através da simulação dos dois cenários descritos na seção anterior (Atual e Verde), foi realizada a validação por análise de sensibilidade, onde, através de modificações controladas nas variáveis de entrada do sistema são verificadas as alterações nas variáveis de saída (BORENSTEIN E BECKER, 2000). A Tabela 1 destaca os valores que foram utilizados de forma constante nas simulações, não sendo modificadas entre um cenário e outro.

Tabela 1 - Taxas Fixas Utilizadas nas Simulações dos Cenários Propostos

\begin{tabular}{|c|c|c|}
\hline Equipamento & Taxa & Valor \\
\hline Servidores & $\begin{array}{l}\text { Média de consumo de } \\
\text { energia } \\
\text { Média de emissão de } \mathrm{CO}^{2} \\
\text { Taxa de aquisição } \\
\text { Taxa de descarte }\end{array}$ & $\begin{array}{l}7446 \text { kwatts } / \text { h por ano (média de } 850 \text { watts } / h^{*} 365 \\
\text { dias do ano * } 24 \text { horas ligado) } \\
4,32 \text { toneladas de } \text { CO }^{2} \text { por ano (considerando o } \\
\text { consumo de } 7446 \text { kwatts } / \mathrm{h} \text { ) } \\
5 \% \\
2,5 \%\end{array}$ \\
\hline PCs & $\begin{array}{l}\text { Média de consumo de } \\
\text { energia } \\
\text { Média de emissão de } \mathrm{CO}^{2} \\
\text { Taxa de aquisição } \\
\text { Taxa de descarte }\end{array}$ & $\begin{array}{l}\mathbf{4 7 , 4 5} \text { kwatts/h por ano (média de } 130 \text { watts } / h^{*} 365 \\
\text { dias do ano. O número de horas ligado é o valor que } \\
\text { será utilizado como variável de simulação ) } \\
\mathbf{0 , 0 3} \text { toneladas de } \mathrm{CO}^{2} \text { por ano (considerando o } \\
\text { consumo de } 47,45 \mathrm{kwatts} / \mathrm{h} \text { ) } \\
10 \% \\
5 \%\end{array}$ \\
\hline Monitores & $\begin{array}{l}\text { Média de consumo de } \\
\text { energia } \\
\text { Média de emissão de } \mathrm{CO}^{2} \\
\text { Taxa de aquisição } \\
\text { Taxa de descarte }\end{array}$ & $\begin{array}{l}\mathbf{9 , 1 2 5} \text { kwatts/h por ano (média de } 25 \text { watts/hora * } \\
365 \text { dias do ano. O número de horas ligado é o valor } \\
\text { que será utilizado como variável de simulação) } \\
\mathbf{0 , 0 1} \text { toneladas de } \mathrm{CO}^{2} \text { por ano (considerando o } \\
\text { consumo de } 9,125 \mathrm{kwatts} / \mathrm{h} \text { ) } \\
10 \% \\
5 \%\end{array}$ \\
\hline
\end{tabular}

Apesar de a universidade possuir um gerador de energia que tem condições de fornecer o total de energia consumido pela Unidade de TI, os técnicos não conseguem identificar as médias de consumo de servidores, PCs e monitores de forma isolada dos demais equipamentos eletrônicos, tais como os eletrodomésticos. Por essa razão, e por tratar-se apenas de uma simulação, as médias de consumo de energia e de emissão de $\mathrm{CO}^{2}$ utilizadas foram retiradas de sites de referência da web. A média de consumo de energia foi calculada com base no site eHow (EHOW, 2015) e a média de emissão de $\mathrm{CO}^{2}$ foi calculada segundo o site Iniciativa Verde (INICIATIVA VERDE, 2015), que disponibiliza uma calculadora onde é possível informar o número de $\mathrm{Kw} / \mathrm{h}$ consumido e como resultado gera o total de $\mathrm{CO}^{2}$ emitido em toneladas.

Os técnicos também não souberam precisar ao certo as taxas médias de aquisição e descarte dos equipamentos, e os valores informados são valores esti-

Revista Produção Online, Florianópolis, SC, v. 16, n. 3, p. 1079-1096, jul./set. 2016. 
mados. Apesar disto, estes valores, por serem fixos e não sofrerem alterações entre os cenários, não irão interferir no resultado final da simulação.

A Tabela 2 detalha os valores utilizados como variáveis de controle em cada uma das simulações, para as taxas que representam as práticas verdes selecionadas.

Tabela 2 - Taxas Variáveis Utilizadas nas Simulações dos Cenários Propostos

\begin{tabular}{lll}
\hline Variável/Cenário & Atual & Verde \\
\hline Taxa de virtualização de servidores & $1 \%$ & $10 \%$ \\
Tempo de monitor ligado & 24 horas & 8 horas \\
Tempo de PC Ligado & 24 horas & 8 horas \\
\hline
\end{tabular}

Os resultados obtidos podem ser visualizados, na forma de gráficos, na Figura 3, referentes ao consumo de energia, e na Figura 4, referentes à emissão de $\mathrm{CO}^{2}$. As figuras demonstram os gráficos gerais do consumo de energia e da emissão de $\mathrm{CO}^{2}$ da Unidade de $\mathrm{TI}$ e depois detalham, separadamente, os resultados de cada um dos equipamentos analisados.

Figura 3 - Comparação dos Cenários quanto ao Consumo de Energia

Atual

Verde

Consumo de Energia

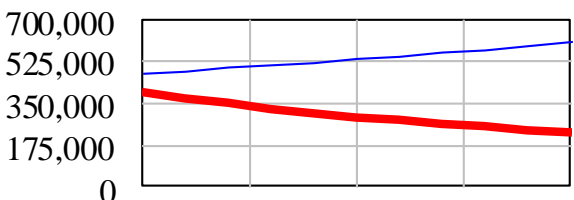

Consumo Monitores

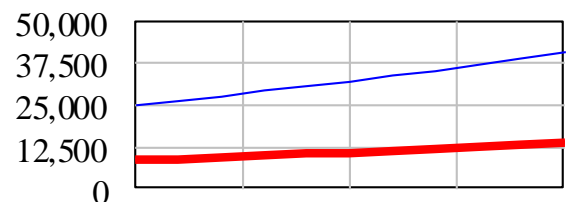

Consumo PC

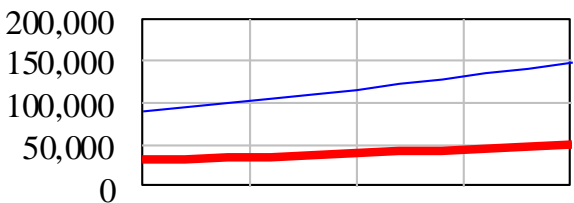

Consumo Servidores

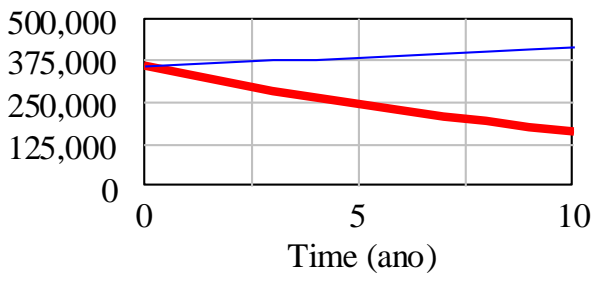

Figura 4 - Comparação dos Cenários quanto a Emissão de $\mathrm{CO}^{2}$

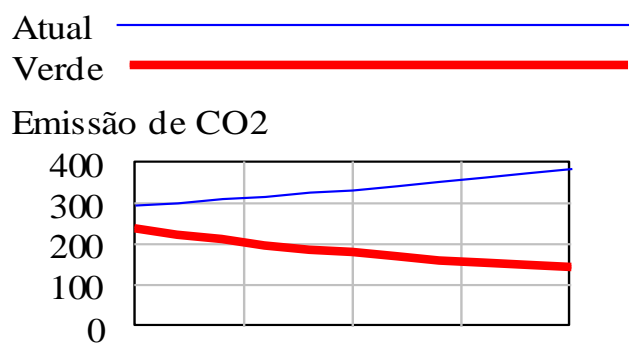

Emissão Monitores

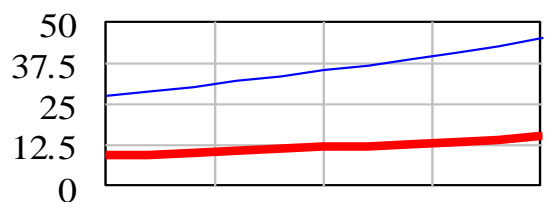

Emissão PC

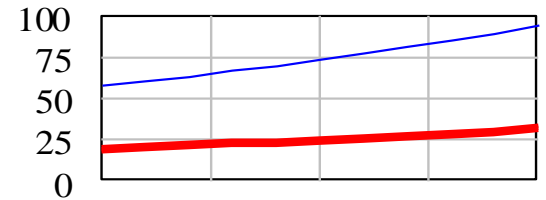

Emissão Servidores

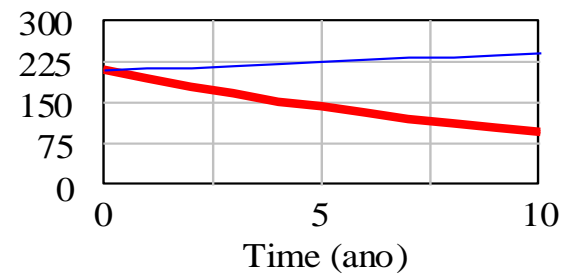


Pode-se verificar que a tendência de consumo de energia e da emissão de $\mathrm{CO}^{2}$ no cenário atual, sem a aplicação de nenhuma prática verde, é de elevação, enquanto que no cenário verde é apresentar uma queda significativa. Comparando os dois cenários percebe-se uma redução de $23 \%$ no consumo de energia e na emissão de $\mathrm{CO}^{2}$ já no primeiro ano de utilização das práticas verdes. Essa redução chega a $45 \%$ no quinto ano e a $62 \%$ ao final dos 10 anos de simulação. A mesma proporção de redução é observada em ambas as taxas, pois o cálculo da quantidade de $\mathrm{CO}^{2}$ emitida é realizado com base no consumo de energia.

Analisando os gráficos por equipamento, percebe-se que, em função das taxas de aquisição tanto de monitores quanto de PCs serem sempre superiores às taxas de descarte, em ambos os cenários há uma tendência de aumento no consumo de energia e de emissão de $\mathrm{CO}^{2}$, porém, com a aplicação de práticas verdes muito simples, pode-se verificar uma redução de até $67 \%$ nas taxas. A redução ocorre em igual proporção nos dois equipamentos em função de que a única variável modificada - tempo ligado - teve o mesmo valor alterado para os dois equipamentos em igual proporção - de 24 horas para 8 horas ligado.

Já no caso dos servidores, no cenário atual, em função da taxa extremamente baixa de virtualização, há a verificação de que em 10 anos o aumento no consumo de energia e na emissão de $\mathrm{CO}^{2}$ possa chegar a $16 \%$. No cenário Verde, com a aplicação de uma taxa de virtualização de apenas $10 \%$ já é possível verificar que as taxas tendem a diminuir ao longo dos 10 anos simulados, verificando $54 \%$ de redução. A comparação entre os cenários demonstra uma redução de $60 \%$ com a utilização da virtualização.

\section{CONSIDERAÇÕES FINAIS}

O impacto ambiental provocado pelo uso das tecnologias de informação está se tornando uma crescente preocupação por meio das empresas e dos governos. A TI Verde engloba desde práticas simples de uso da TI até processos elaborados de fabricação e descarte.

Este trabalho buscou por meio de simulação computacional demonstrar os ganhos ambientais e financeiros que podem ser obtidos pela Unidade de TI de uma universidade através da utilização de práticas simples da TI Verde: virtualização de servidores e desligamento de equipamentos quando ociosos. Como resultado atingiu-se, num horizonte de 10 anos, uma economia de até $62 \%$ no consumo de energia e na emissão de $\mathrm{CO}^{2}$. Apesar dos resultados tenderem muito para a obviedade no que tange a questão de economia energética, entende-se como atingidos os objetivos deste estudo que, antes de qualquer coisa, era validar o uso de simulação computacional como forma de auxiliar o processo decisório para a adoção ou não de práticas da TI Verde.

Como restrições da pesquisa destacam-se que foram ignoradas questões como a refrigeração do data center que também possui grande impacto no consumo de energia, e quanto ao tipo de monitores e PCs disponíveis, sabendo que aqueles 
que possuem a função de economia de energia e que hibernam quando estão fora de uso tem um consumo médio de energia diferente do que foi considerado nesse trabalho.

Como trabalhos futuros, sugere-se que seja desenvolvido um novo estudo, que não seja simulação, mas sim um caso real, onde o consumo de energia seja medido no inicio do estudo e sejam realizadas diversas ações verdes, durante um ano, incluindo-se a conscientização dos funcionários, e após esse ano seja realizada nova medição para verificar os benefícios obtidos.

\section{REFERÊNCIAS}

ANDRADE, E, L. de. Introdução à pesquisa operacional: métodos e modelos para a análise de decisão. 4. ed. Rio de Janeiro: LTC, 4. Ed, 2011.

ARNFALK, P.; PILEROT, U.; SCHILLANDER, P.; GRÖNVAL, P. Green IT in practice: virtual meetings in Swedish public agencies. Journal of Cleaner Production. 2015. In Press. http://dx.doi.org/10.1016/j.jclepro.2015.08.063.

BORENSTEIN, D; BECKER, J. L. Validating Decision Support Systems. In: KENT, A.; WILLIAMS, J. G. (Eds.), Encyclopedia of Microcomputers. New York: Marcel Dekker, 2000.

CHUANG, S.P.; HUANG, S.J. Effects of Business Greening and Green IT Capital on Business Competitiveness. Journal of Business Ethics. V.128, n.1, p. 221 -231, abr. 2015. http://dx.doi.org/10.1007/s10551-014-2094-y

CHOU, D.C. Risk Identification in Green IT Practice. Computer Standars \& Interfaces. v.35, n. 2, p. 231-237, 2013. http://dx.doi.org/10.1016/j.csi.2012.10.001

CHOU, D.C.; CHOU, A.Y. Awareness of Green IT and its value model. Computer Standard and Interfaces, 34, p. 447-451. 2012. http://dx.doi.org/10.1016/j.csi.2012.03.001

COOPER, V.A., MOLLA, A. Absorptive Capacity and Contextual Factors that Influence Green IT Assimilation. Australasian Journal of Information Systems, v.18, n.3, 2014. http://dx.doi.org/10.3127/ajis.v18i3.1099

DIAS, G.F., ET AL. Práticas organizacionais ambientalmente corretas relacionadas com a Tecnologia de Informação: um estudo qualitativo em universidades brasileiras bem ranqueadas. XXXVII EnAnpad, 2013.

EHOW, Disponível em: <http://www.ehow.com/info_8763694_much-computer-use-perhour.html>. Acesso em: Abr. 2015.

FARIA, A.C.; MARTINS, M.S., SIQUEIRA, L.D. TI verde: mito ou realidade na indústria digital brasileira. XXXVII EnAnpad. 2013.

FAUCHEUX, S.; NICOLAÏ, I. IT for green and green IT: A proposed typology of ecoinnovation . Ecological economics, v.70, n. 11, p. 2020 -2027, 2011.

http://dx.doi.org/10.1016/j.ecolecon.2011.05.019

FORRESTER, J. W. Industrial dynamics. The MIT Press, 1961 
FREITAS FILHO, P. J. Introdução à modelagem e simulação de sistemas. 2. ed. Florianópolis: Visual Books, 2008.

INICIATIVA VERDE, Disponível em: <http://www.iniciativaverde.org.br/pt/calculadora>. Acesso em: Abr. 2015.

JAYO, Martin; VALENTE, Rafael. POR UMA TI MAIS VERDE. GVexecutivo. v.9, n. 1, jan./jun. 2010.

LUNARDI, G.L.; FRIO, R. S.; BRUM, M. M. Tecnologia da Informação e Sustentabilidade: Um estudo sobre a disseminação das práticas de TI Verde nas organizações. XXXV Encontro da Anpad - EnAnpad, 2011.

LUNARDI, G.L.; ALVES, A.P.F.; SALLES, A.C. Desenvolvimento de uma escala para avaliar o grau de utilização da tecnologia da informação verde pelas organizações R.Adm., São

Paulo, v.49, n.3, p.591-605, jul./set. 2014. http://dx.doi.org/10.5700/rausp1170

LUNARDI, G.L.; DOLCI, D.B.; SALLES, A.C. Antecedentes e consequentes da adoção da ti verde nas organizações: um estudo sobre o papel das ações organizacionais e o seu impacto no desempenho ambiental e na imagem corporativa. XXXIX ENANPAD. 2015.

MURUGESAN, San. Harnessing green it: principles and practices, IT Professional, v. 10, n.1, p. 24-33, 2008. http://dx.doi.org/10.1109/mitp.2008.10

NASCIMENTO, R.L. ET AL. Estudo de modelagem e simulação de uma fila em uma empresa salineira. XXXIV ENEGEP, 2014.

NETO, H. G.; OLIVEIRA, J. B.; ANDRADE , C. R. F. Modelagem e simulação computacional em uma empresa de confecção de roupas: um estudo sobre o desempenho de um processo produtivo. XXXIV ENEGEP, 2014.

PARAÍSO, M. R. A.; SOARES, T. O. R.; ALMEIDA, L. A. Desafios e práticas para a inserção da Tecnologia da Informação Verde nas empresas baianas: um estudo sobre a perspectiva dos profissionais de Tecnologia da Informação. RGSA - Revista de Gestão Social e Ambiental, v.3, n. 3, p. 85-101, 2009. http://dx.doi.org/ 10.5773/rgsa.v3i3.178

PAPPEN, S.A.; YONENAGA, W.H. O setor de piscicultura no mato grosso: uma abordagem pela dinâmica de sistemas. XXXIV ENEGEP, 2014.

PIDD, M. Modelagem empresarial: ferramentas para tomada de decisão. Ed. Bookman, 1998.

RAGSDALE, C.T. Modelagem e análise de decisão. São Paulo: CENGAGE Learning. 2009.

ROSSONI, L. Modelagem e Simulação Soft em Estratégia. Revista Produção on-line, v. 6, n. 2, 2006. http://dx.doi.org/ 10.14488/1676-1901.v6i2.291

SALLES , A. C.; ALVES, A. P. F.; DOLCI, D. B.; LUNARDI, G. L. Adoção de Práticas de TI Verde nas Organizações: Um Estudo Baseado em Mini Casos. IV Encontro da Administração da Informação - EnADI - 2013.

SEBRAE. TI Verde: conceitos e práticas. Disponível em:

$<$ http://www.sebrae.com.br/sites/PortalSebrae/artigos/TI-Verde:-conceitos-e-

pr\%C3\%A1ticas>. Acesso em: Abr. 2015.

Revista Produção Online, Florianópolis, SC, v. 16, n. 3, p. 1079-1096, jul./set. 2016. 
SOUZA, M. A. S.; SILVA, D. N. Validação de um Instrumento para avaliar a utilização de práticas da TIV. XXXIII ENEGEP. 2013.

UDDIN, M.; RAHMAN, A.A. Server consolidation: an approach to make data centers energy efficient \& green. International Journal of Scientific \& Engineering Research, v 1, n 1, Out. 2010

VENTANA SYSTEMS. Vensim Simulation Software. Disponível em: <http://www.vensim.com>. Acesso em: mar. 2015.

\section{() $(1)$}

Artigo recebido em 26/01/2016 e aceito para publicação em 10/03/2016

DOI: http://dx.doi.org/ 10.14488/1676-1901.v16i3.2333 